\title{
Antimicrobial Compound from Endophytic Pseudomonas azotoformans UICC B-91 of Neesia altissima (Malvaceae)
}

\author{
Rina Hidayati Pratiwi ${ }^{1,2, *}$, Eva Oktarina ${ }^{3}$, Wibowo Mangunwardoyo ${ }^{4}$, Iman Hidayat ${ }^{5}$, Endang Saepudin ${ }^{6}$
}

\author{
Rina Hidayati Pratiwi ${ }^{1,2, *}$, \\ Eva Oktarina ${ }^{3}$, Wibowo \\ Mangunwardoyo $^{4}$, Iman Hidayat ${ }^{5}$, \\ Endang Saepudin 6
}

${ }^{1}$ Department of Mathematics and Natura Sciences Education, Faculty of Post Graduated, Universitas Indraprasta PGRI, South Jakarta, INDONESIA.

${ }^{2}$ Department of Biological Education, Faculty of Mathematics and Natural Sciences, Universitas Indraprasta PGRI, East Jakarta, INDONESIA.

${ }^{3}$ Master Program of Chemistry, Faculty of Mathematics and Natural Sciences, Universitas Indonesia, Depok, INDONESIA. ${ }^{4}$ Department of Biology, Faculty of Mathematics and Natural Sciences, Universitas Indonesia, Depok, INDONESIA ${ }^{5}$ Research Center for Biomaterial, Indonesian Institute of Sciences (LIPI), Cibinong, INDONESIA.

${ }^{6}$ Department of Chemistry, Faculty of Mathematics and Natural Sciences, Universitas Indonesia, Depok, INDONESIA.

\section{Correspondence}

\section{Rina Hidayati Pratiwi}

Department of Mathematics and Natural Sciences Education, Faculty of Post

Graduated, Universitas Indraprasta PGRI South Jakarta; Department of Biological Education, Faculty of Mathematics and

Natural Sciences, Universitas Indraprasta

PGRI, East Jakarta, INDONESIA

Phone no: +628176301017

E-mail: rina.hp2012@gmail.com

History

- Submission Date: 23-11-2021;

- Review completed: 07-12-2021;

- Accepted Date: 15-12-2021.

DOI : 10.5530/pj.2022.14.23

Article Available online http://www.phcogj.com/v14/i1

\section{Copyright}

(C) 2022 Phcogj.Com. This is an openaccess article distributed under the terms of the Creative Commons Attribution 4.0 International license.

\section{ABSTRACT}

Centrifugated extract of endophytic Pseudomonas azotoformans strain UICC B-91 has been observed for its antimicrobial activity against Escherichia coli ATCC 8739, Bacillus cereus ATCC 10876, Staphylococcus aureus ATCC 6583, Staphylococcus aureus ATCC 25923, Salmonella typhimurium ATCC 25241, Pseudomonas aeruginosa ATCC 15442, Bacillus subtilis ATCC 19659 and Candida albicans ATCC 10231, with minimum concentration in the range $250 \mu \mathrm{g} / \mathrm{mL}$. But, the novel compound of the bioactive substances which relevant for antimicrobial activity have not been described. Further fractionation and identification of the extract by thin layer chromatography (TLC) and liquid chromatography mass spectrophotometry (LCMS-MS) detected several compound as futoamide $\left(\mathrm{C}_{18} \mathrm{H}_{23} \mathrm{NO}_{3}\right)$, gentialutine $\left(\mathrm{C}_{9} \mathrm{H}_{11} \mathrm{NO}\right)$, gentiabetine $\left(\mathrm{C}_{9} \mathrm{H}_{11} \mathrm{NO}_{2}\right)$, 1-[(2E,4E)-2,4-decadienoyl]pyrrolidine $\left(\mathrm{C}_{14} \mathrm{H}_{23} \mathrm{NO}\right)$, lycopodine $\left(\mathrm{C}_{16} \mathrm{H}_{25} \mathrm{NO}\right)$ and dihydrolycopodine $\left(\mathrm{C}_{16} \mathrm{H}_{27} \mathrm{NO}\right)$. The majority compounds containing $\mathrm{CHON}$ elements with molecular weight approximately $100-450 \mathrm{Da}$.

Keywords: Alkaloid, Antimicrobial compound, Extraction, P. azotoformans.

\section{INTRODUCTION}

P. azotoformans strain UICC B-91 was isolated from Nessia altissima Blume, traditional medicinal plant endemic from Indonesia ${ }^{1}$ that effective against gonorrhoeae ${ }^{2}$ due to Neisseria gonorrhoeae infection. But there is no publication research on bioactive natural compound from N. altissma, yet. Endophytic microorganism has genetic diversity due to adaption to host environment and might be transfer gen from host, that relate to the bioactive compound produced. ${ }^{3}$ Previous research has been observed the antimicrobial activity from centrifugated extract of endophytic P. azotoformans, against several bacteria and C. albicans. ${ }^{4}$ with notice result on its anticandidal activity. For further observation, the antimicrobial compounds were identified by mass spectrophotometry.

Several antimicrobial compounds which have been identified from $P$. azotoformans was rhamnolipid that able to inhibit various species of Bacillus, ${ }^{5}$ 1-acetyl-beta-carboline that able to inhibit MRSA, ${ }^{6}$ 2-aminofenol, 5-methyl-2,4-imidazolidinedione, 1,4-benzenediamine,N-(1-methylethyl)-N-phenyl, pirrol[1,2-a]pirazin-1,4-diona, pirrol[1,2-a] pirazin-1,4-diona,hexahydro-3-(phenylmethyl), and hexadecenoic acid that able to inhibit Neisseria meningitidis and S. aureus. ${ }^{7}$ Thus research encourage us to explore antimicrobial compound especially alkaloids. Alkaloid exhibit broad bioactivities, potentially new type of broad spectrum natural antimicrobial compound, and low tendency to produce resistance. ${ }^{8,9}$

This study sought to explore antimicrobial activity on extract of $P$. azotoformans without centrifugation on extraction and observed the chemical compound from endophytic $P$. azotoformans as antimicrobial compound.

\section{MATERIALS AND METHODS}

\section{Microorganisms}

P. azotoformans strain UICC B-91 used in this research was deposit culture of Universitas Indonesia of Culture Collection. Microorganisms for antibacterial susceptibility screening were $E$. coli ATCC 8739 , B. cereus ATCC 10876, B. subtilis ATCC 19659, P. aeruginosa ATCC 15442, S. typhimurium ATCC 25241, S. aureus ATCC 6583, S. aureus ATCC 25923, and C. albicans ATCC 10231.

\section{Extraction of antimicrobial compound}

The extractions were proceeded by two culture mediums as Nutrient Broth (NB) and Rehydrated Broth (RB). The RB compositions are yeast extract, peptone, and $\mathrm{MgSO}_{4}$. Fermentations and various extractions treatments were described on Figure 1. The extracts then concentrated by rotary evaporator on $40^{\circ} \mathrm{C}$. The resulted was crude extract (Table 1). Crude extract then separated through column chromatography using silica gel 60 G F254.

\section{Antimicrobial activity}

Antimicrobial activity analysis were regarding to disc diffusion. ${ }^{10,11}$ The antimicrobial activity was measured by diameter of clear zone minus diameter of disc diffusion.

\section{Thin Layer Chromatography (TLC)}

TLC analytical, TLC-bioautography and semipreparative separation were performed on aluminium silica TLC F254 plates. The TLC plates were developed with mobile phase chloroform: methanol (9:1) (F1, F3, F4, and F5) and hexane: ethyl acetate (7:3) (F2). Screening for antimicrobial compound conducted by TLC-bioautography. ${ }^{12}$ Positive result reflected on clear zone developed by Rf. Further TLC-separation on clear zone-Rf were developed with chloroform: ethyl acetate (5:5) (F1, F4, and F5). 


\section{Table 1. Description of the research.}

\begin{tabular}{llll} 
Extract & $\begin{array}{l}\text { Antimicrobial } \\
\text { activity }\end{array}$ & $\begin{array}{l}\text { Structure } \\
\text { identification }\end{array}$ & Fraction \\
\hline RB.CS.EA & Ref in press $^{4}$ & n.d. & \\
RB.CS.CF & Ref in press $^{4}$ & n.d. & \\
RB.CS.DCM & Ref in press $^{4}$ & This publication & F1, F2 \\
RB.CP.EA & Ref in press $^{4}$ & This publication & F3 \\
RB.NC.EA & This publication $^{\text {This publication }}$ & F4 \\
NB.NC.EA & This publication & This publication & F5 \\
n.d. $=$ not determined & &
\end{tabular}

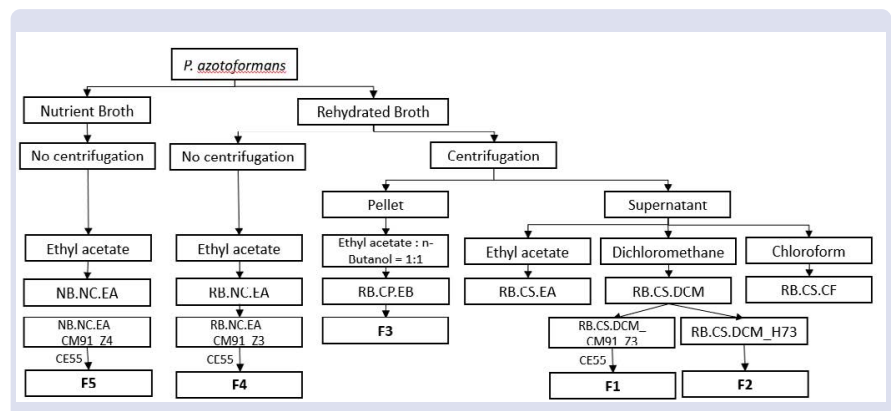

Figure 1. Variation of treatment on P. azotoformans extracts.

\section{Identification and structure determination}

The analysis on fractions was performed by LCMS/MS using XEVO G2-XS QT of mass spectrophotometer. The ionisation was using Electro Spray Ionisation (ESI), with scan range 50 to $1,200 \mathrm{~m} / \mathrm{z}$. Ultraperformance liquid chromatography (UPLC) was performed using a Waters Acquity UPLC I-Class system. Chromatographic separation was caried out with ACQUITY UPLC ${ }^{\otimes}$ BEH C8 $(2.1 \times 100 \mathrm{~mm}, 1.7$ $\mu \mathrm{m})$ on $40^{\circ} \mathrm{C}$. The mass-to-charge ratio $(\mathrm{m} / \mathrm{z})$ values and retention time analysed was identified using UNIFI software. For candidate mass, the values were analysed using databases in Metfrag and PubChem. The identified compounds were also compared using Pseudomonas aeruginosa Metabolome Database by University of Maryland School of Pharmacy (PAMDB), Natural Product Activity and Species Source Database (NPASS), and The Dictionary of Alkaloid. ${ }^{13}$ The ${ }^{13} \mathrm{C}-\mathrm{NMR}$ was performed by NMR Agilent with DD2 system console and $125 \mathrm{MHz}$ reading. Chloroform was used as solvent (DDCl3) and tetramethylsilane (TMS) as chemical shifting reference.

\section{RESULT}

\section{Antimicrobial activity on crude extract}

The result of the antimicrobial activity of ethyl acetate extract of P. azotoformans endophytic are described on Table 2. The in vitro antimicrobial activity was shown on both crude ethyl acetate extract on gram negative bacteria as E. coli ATCC 8739, S. typhimurium ATCC 25241, $P$. aeruginosa ATCC 15442; gram positive bacteria as $B$. subtilis ATCC 19659, B. cereus ATCC 10876, S. aureus ATCC 25923, S. aureus ATCC 6583; and yeast of $C$. albicans ATCC 10231. The range of minimum inhibition concentration of NB.NC.EA was on 50,000 $-5,000 \mu \mathrm{g} / \mathrm{mL}$ and for RB.NC.EA, it goes lower until $1,000 \mu \mathrm{g} / \mathrm{mL}$. Crude extract of RB.NC.EA also has widest clear zone diameter than NB.NC.EA, especially on P. aeruginosa and C. albicans. The minimum inhibition concentration of C. albicans on RB.NC.EA was $1,000 \mu \mathrm{g} / \mathrm{mL}$. NB.NC.EA was better on inhibiting growth of gram-positive bacteria than gram-negative bacteria. Tetracycline $(100 \mu \mathrm{g} / \mathrm{mL})$ as broad range antibiotic was given clear zone as positive result of inhibition on gram positive and negative bacteria. Ketoconazole $(10,000 \mu \mathrm{g} / \mathrm{mL})$ as antifungal was given clear zone as positive result of inhibition on $C$. albicans. RB.NC.EA exhibit widest clear zone than ketoconazole for $C$. albicans on concentration $10,000 \mu \mathrm{g} / \mathrm{mL}$.

\section{Separation and TLC-bioautography}

The crude extracts were separated through chromatography column with chloroform-methanol in gradual concentration. The positive result on TLC-bioautography was shown on fractionation CM91. The positive Rf on RB.NC.EA_CM91 were on $0.8625,0.7625$, and 0.6625 . The significant clear zone was shown on Rf 0.7625 (Figure 2). For NB.NC.EA, the Rf was on 0.7125 (picture not shown). The fractionation CM91 of extract RB.NC.EA and NB.NC.EA were further separated with mobile phase CE55.

\section{Antimicrobial activity on fraction}

Fraction of RM.NC.EA (F4) able to inhibit C. albicans and bacteria. For NB.NC.EA (F5), it also able to inhibit $C$. albicans and bacteria, except S. aureus ATCC 6583. Both of fractions have inhibition concentration in the range 1,000 - $250 \mu \mathrm{g} / \mathrm{mL}$. F4 and $\mathbf{F} 5$ has shown lower inhibition concentration than the crude extract, except $S$. aureus ATCC 6583 on F5. Generally, F4 and F5 have better inhibition on gram-negative bacteria than positive. While on crude extract the RB.NC.EA was shown wide diameter clear zone on $P$. aeruginosa and $C$. albicans, the fraction (F4) was giving similar range diameter $(2.00-4.00 \mathrm{~mm})$ to all pathogenic microbe tested.

\section{Identification of fractions}

The result on identification exhibits the fractions were not single compound. The result on identification by LCMS/MS were describe on table 4 , the structure of identified compound was on figure 3 , and chromatogram was on figure 4. F1 and F4 further separation were conduct by TLC-separation with chloroform-ethyl acetate $(5: 5)$ and gave cumulation on time retention $9-10$ minutes. For F2 and F3, without further separation, the peak was also detected on time retention 5 and 8 minutes. The mass spectrophotometry was using ion $\mathrm{H}^{+}$and $\mathrm{Na}^{+}$as ion adduct.

\section{Futoamide (1)}

Futoamide was detected on $\mathbf{F} \mathbf{1}$ and $\mathbf{F} 4$. Futoamide given protonated molecular ion at $\mathrm{m} / \mathrm{z}[\mathrm{M}+$ ion adduct $] 324.1569$, ion ${ }^{+} \mathrm{Na}$ was used as adduct ion, the neutral mass 301.16779 for which molecular formula $\mathrm{C}_{18} \mathrm{H}_{23} \mathrm{NO}_{3}$ was generated. The compound was tentatively identified as futoamide. The fragmentation on dioxole ring of this compound resulted in the presence of ions at $\mathrm{m} / \mathrm{z} 272.16415[\mathrm{M}-\mathrm{CHO}]^{++}$, and 122.05819 $\left[\mathrm{M}-\mathrm{C}_{10} \mathrm{H}_{12}-\mathrm{H}_{2} \mathrm{O}-\mathrm{CHO}\right]^{+*}$. The fragmentation on aliphatic resulted in the presence of ion at $\mathrm{m} / \mathrm{z} 149.05952\left[\mathrm{M}-\mathrm{C}_{3} \mathrm{H}_{9}-\mathrm{CNC}(\mathrm{O})-\mathrm{C}_{4} \mathrm{H}_{5}\right]^{0+}$.

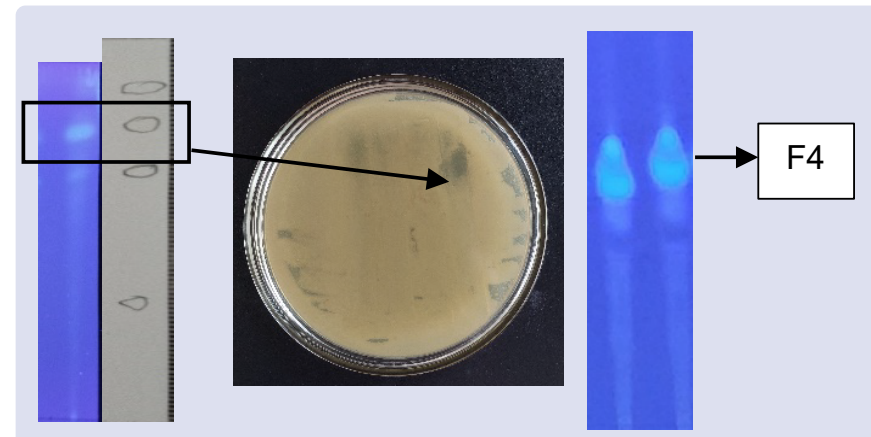

Figure 2. TLC-Bioautography of extract RM.NC.EA with CM91 as mobile phase on C. albicans (A and B) further separation with CE55 develop F4 (C). 
<smiles>CC(C)(C)NC(=O)/C=C/CC/C=C/c1ccc2c(c1)OCO2</smiles>

1

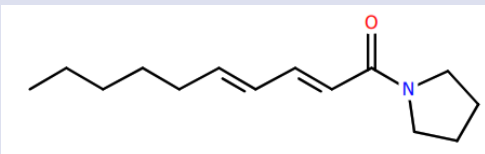

4

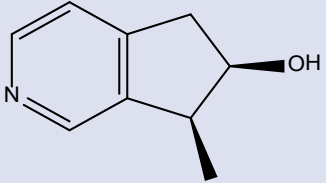

2

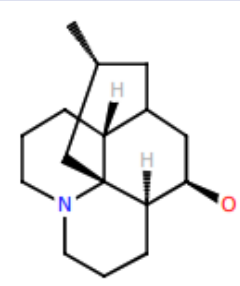

5

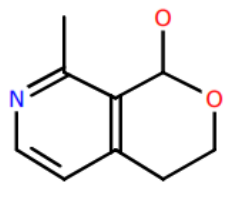

3

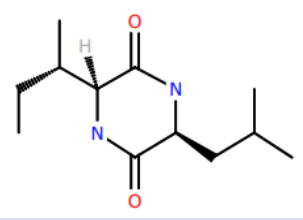

7

Figure 3. Structures of antimicrobial compounds from endophytic P. azotoformans.

(1)

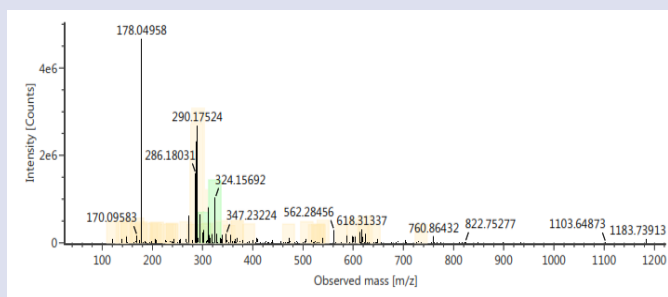

(2)

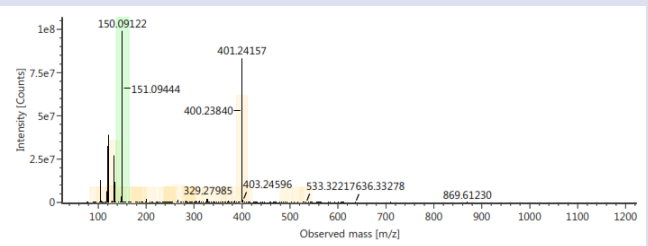

(3)

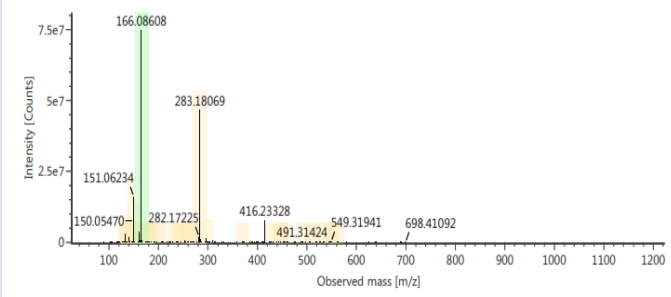

(4)

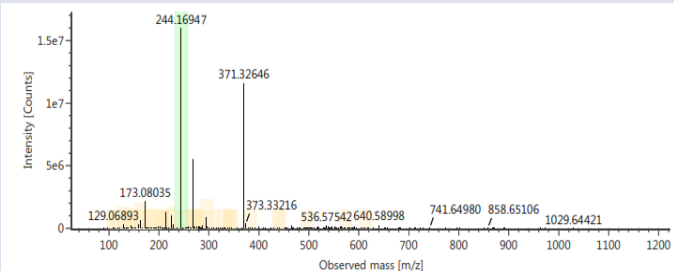

(5)

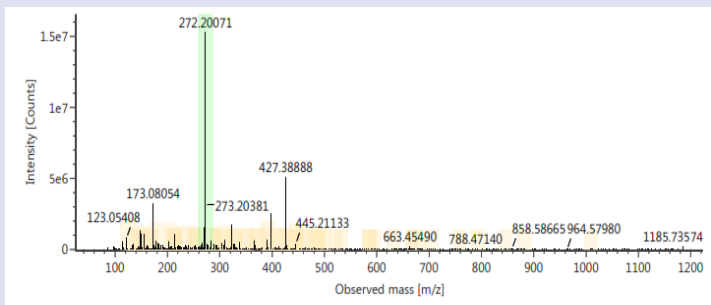

(6)

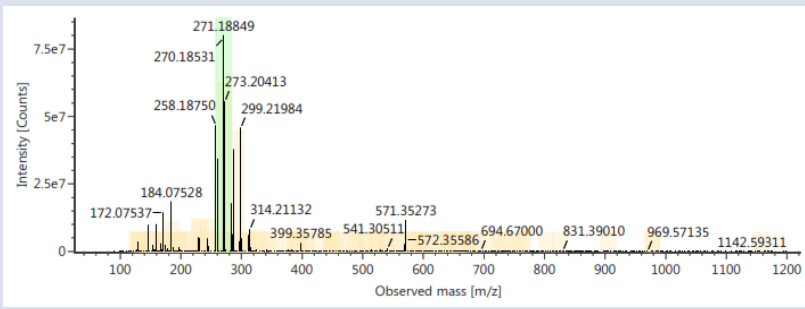

(7)

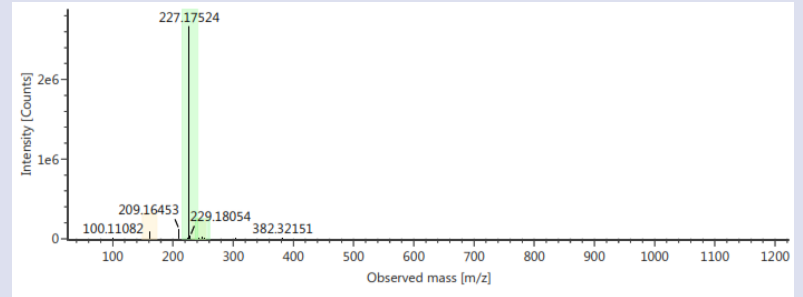

Figure 4. Chromatograms of antimicrobial compound from endophytic P. azotoformans. 
Table 2. In vitro antibacterial activity on ethyl acetate extract of $P$. azotoformans using agar diffusion method.

\begin{tabular}{|c|c|c|c|c|c|c|c|c|}
\hline \multirow{2}{*}{$\begin{array}{l}\text { Conc.. } \\
(\mu \mathrm{g} / \mathrm{mL})\end{array}$} & \multicolumn{8}{|c|}{ Microorganism } \\
\hline & $\begin{array}{l}\text { S. aureus } \\
\text { ATCC } 6583\end{array}$ & $\begin{array}{c}\text { S. aureus } \\
\text { ATCC } 25923\end{array}$ & $\begin{array}{l}\text { S. typhimurium } \\
\text { ATCC } 25241\end{array}$ & $\begin{array}{c}\text { E. coli } \\
\text { ATCC } 8739\end{array}$ & $\begin{array}{l}\text { P. aeruginosa } \\
\text { ATCC } 15442\end{array}$ & $\begin{array}{c}\text { B. cereus } \\
\text { ATCC } 10876\end{array}$ & $\begin{array}{l}\text { B. subtilis } \\
\text { ATCC } 19659\end{array}$ & $\begin{array}{l}\text { C. albicans } \\
\text { ATCC } 10231\end{array}$ \\
\hline \multicolumn{9}{|c|}{ NB.NC.EA } \\
\hline 50,000 & $3.33 \pm 0.56$ & $2.50 \pm 0.71$ & $2.00 \pm 0.00$ & $1.33 \pm 0.58$ & $2.00 \pm 0.50$ & $2.00 \pm 0.00$ & $2.50 \pm 0.71$ & $5.50 \pm 1.50$ \\
\hline 10,000 & $1.50 \pm 0.00$ & $1.00 \pm 0.00$ & $2.00 \pm 0.00$ & $2.33 \pm 0.58$ & $2.00 \pm 0.00$ & $2.00 \pm 0.00$ & $1.00 \pm 0.00$ & $3.00 \pm 0.71$ \\
\hline 5,000 & - & - & $2.00 \pm 0.00$ & $1.83 \pm 0.76$ & - & $1.00 \pm 0.00$ & - & $1.50 \pm 0.50$ \\
\hline 1,000 & - & - & - & - & - & - & - & - \\
\hline 100 & - & - & - & - & - & - & - & - \\
\hline \multicolumn{9}{|c|}{ RM.NC.EA } \\
\hline 50,000 & $4.00 \pm 0.00$ & $4.50 \pm 0.71$ & $4.50 \pm 1.00$ & $3.50 \pm 0.71$ & $14.50 \pm 0.12$ & $3.00 \pm 1.00$ & $3.00 \pm 0.00$ & $13.25 \pm 0.35$ \\
\hline 10,000 & $4.00 \pm 0.00$ & $3.00 \pm 0.00$ & $4.00 \pm 0.00$ & $5.67 \pm 1.76$ & $4.00 \pm 0.00$ & $1.00 \pm 0.00$ & $1.50 \pm 0.71$ & $11.50 \pm 0.71$ \\
\hline 5,000 & $1.50 \pm 0.00$ & & $4.00 \pm 0.00$ & $3.50 \pm 0.87$ & $3.75 \pm 0.35$ & $6.00 \pm 0.00$ & $2.75 \pm 1.06$ & $8.50 \pm 0.87$ \\
\hline 1,000 & - & - & $2.00 \pm 0.00$ & - & - & - & $1.00 \pm 0.00$ & $1.00 \pm 0.00$ \\
\hline 100 & - & - & - & - & - & - & - & - \\
\hline \multicolumn{9}{|l|}{ Control } \\
\hline $\mathrm{C}+$ & $10.00 \pm 1,41$ & $8.50 \pm 0,00$ & $8.75 \pm 0.35$ & $4.50 \pm 0.00$ & $9.00 \pm 0.00$ & $4.50 \pm 0.70$ & $11.00 \pm 0.71$ & $4.50 \pm 1.41$ \\
\hline C - & - & - & - & - & - & - & - & - \\
\hline
\end{tabular}

Result: Diameter of clear zone \pm standard deviation $(\mathrm{mm})$

Table 3. In vitro antimicrobial activity on fraction of $P$. azotoformans using agar diffusion method.

\begin{tabular}{|c|c|c|c|c|c|c|c|c|}
\hline \multirow{2}{*}{$\begin{array}{l}\text { Cons. } \\
(\mu \mathrm{g} / \mathrm{mL})\end{array}$} & \multicolumn{8}{|c|}{ Microorganism } \\
\hline & $\begin{array}{l}\text { S. aureus } \\
\text { ATCC } 6583\end{array}$ & $\begin{array}{c}\text { S. aureus } \\
\text { ATCC } 25923\end{array}$ & $\begin{array}{l}\text { S. typhimurium } \\
\text { ATCC } 25241\end{array}$ & $\begin{array}{c}\text { E. coli } \\
\text { ATCC } 8739\end{array}$ & $\begin{array}{l}\text { P. aeruginosa } \\
\text { ATCC } 15442\end{array}$ & $\begin{array}{c}\text { B. cereus } \\
\text { ATCC } 10876\end{array}$ & $\begin{array}{l}\text { B. subtilis } \\
\text { ATCC } 19659\end{array}$ & $\begin{array}{l}\text { C. albicans } \\
\text { ATCC } 10231\end{array}$ \\
\hline \multicolumn{9}{|c|}{ NB.NC.EA_Zone 4 (F5) } \\
\hline 1,000 & - & $1.00 \pm 0.00$ & $3.50 \pm 0.00$ & $3.00 \pm 0.00$ & $4.00 \pm 0.00$ & $3.00 \pm 0.00$ & $1.50 \pm 0.00$ & $4.00 \pm 0.00$ \\
\hline 500 & - & $1.00 \pm 0.00$ & $1.00 \pm 0.00$ & $2.33 \pm 0.71$ & $1.50 \pm 0.00$ & $2.00 \pm 0.00$ & - & $1.00 \pm 0.00$ \\
\hline 250 & - & - & $1.00 \pm 0.00$ & $1.00 \pm 0.00$ & - & - & - & - \\
\hline 125 & - & - & - & - & - & - & - & - \\
\hline 62.5 & - & - & - & - & - & - & - & - \\
\hline 31.25 & - & - & - & - & - & - & - & - \\
\hline 15.625 & - & - & - & - & - & - & - & - \\
\hline \multicolumn{9}{|c|}{ RM.NC.EA_Zone 3 (F4) } \\
\hline 1,000 & $4.00 \pm 0.00$ & $4.00 \pm 0.00$ & $4.00 \pm 0.00$ & $3.00 \pm 0.00$ & $3.00 \pm 0.00$ & $4.00 \pm 0.00$ & $4.00 \pm 0.00$ & $3.00 \pm 0.00$ \\
\hline 500 & $3.00 \pm 0.00$ & - & $3.00 \pm 0.00$ & $2.50 \pm 0.00$ & $2.50 \pm 0.00$ & $3.00 \pm 0.00$ & $3.00 \pm 0.00$ & $2.00 \pm 0.00$ \\
\hline 250 & - & - & $2.00 \pm 0.00$ & $2.00 \pm 0.00$ & - & - & - & - \\
\hline 125 & - & - & - & - & - & - & - & - \\
\hline 62.5 & - & - & - & - & - & - & - & - \\
\hline 31.25 & - & - & - & - & - & - & - & - \\
\hline 15.625 & - & - & - & - & - & - & - & - \\
\hline \multicolumn{9}{|l|}{ Control } \\
\hline $\mathrm{C}+$ & $8.50 \pm 0.00$ & $6.50 \pm 0.00$ & $5.50 \pm 0.00$ & $5.00 \pm 0.00$ & $7.50 \pm 0.00$ & $4.50 \pm 0.00$ & $4.50 \pm 0.00$ & $4.50 \pm 0.71$ \\
\hline C - & - & - & - & - & - & - & - & - \\
\hline
\end{tabular}

\section{Gentialutine (2)}

The compounds identified on F2 were Gentialutine (alkaloid) and Gentiatibetine. Gentialutine or (6R,7S)-7-methyl-6,7-dihydro-5Hcyclopenta[c]pyridin-6-ol forming protonated molecular ion $[\mathrm{M}+\mathrm{H}]^{+}$ at $\mathrm{m} / \mathrm{z} 150.0912$ with formula $\mathrm{C}_{9} \mathrm{H}_{11} \mathrm{NO}$. The fragmentation 135.06681 [M- $\left.\mathrm{CH}_{2}\right]^{\cdot+}$ was obtained from the fragmentation on methyl group; $120.08049[\mathrm{M}-\mathrm{CHO}]^{\cdot+}$ was obtained from the cleavage on cyclo-pentane ring which led the loss on one carbon bond to oxygen; further loss on carbon led the formation of ion 107.07233 [M- $\left.\mathrm{C}_{2} \mathrm{H}_{2} \mathrm{O}\right]^{\cdot+}$ and 106.06481 $\left[\mathrm{M}-\mathrm{CH}_{2}-\mathrm{CHO}\right]^{++}$; further loss on another carbon led the formation of ion $94.06481\left[\mathrm{M}-\mathrm{C}_{2} \mathrm{H}_{2}-\mathrm{COH}\right]^{\cdot+}$ and $92.05121\left[\mathrm{M}-\mathrm{C}_{3} \mathrm{H}_{5} \mathrm{O}\right]^{\cdot+}$.

\section{Gentiatibetine (3)}

Gentiatibetine with formula $\mathrm{C}_{9} \mathrm{H}_{11} \mathrm{NO}_{2}$ detected on peak ion $\mathrm{m} / \mathrm{z}$ 165.07898. The fragmentation on the methyl group develops an ion $\mathrm{m} / \mathrm{z} 151.06236\left[\mathrm{M}-\mathrm{CH}_{2}\right]^{\cdot+}$ and $150.05470\left[\mathrm{M}-\mathrm{CH}_{3}\right]^{\cdot+}$; fragmentation on alcohol group develops ion $\mathrm{m} / \mathrm{z} 148.07529[\mathrm{M}-\mathrm{OH}]^{++}$, further fragmentation with one carbon develop ion $\mathrm{m} / \mathrm{z} 136.07425$ [M- CHO] ${ }^{\cdot+}$ and $134.05973\left[\mathrm{M}-\mathrm{CH}_{2}-\mathrm{OH}\right]^{+*}$; ion $\mathrm{m} / \mathrm{z} 123.06763\left[\mathrm{M}-\mathrm{C}_{2} \mathrm{H}_{2} \mathrm{O}\right]^{++}$; fragmentation on pyran ring develop ion $\mathrm{m} / \mathrm{z} 122.09581\left[\mathrm{M}-\mathrm{CO}_{2}\right]^{\cdot+}$ with the rearrangement of hydrogen and $120.08035\left[\mathrm{M}-\mathrm{CHO}_{2}\right]^{*+}$ the formation of carbocyclic acid; further fragmentation develop ion $\mathrm{m} / \mathrm{z}$ 107.07164 $\left[\mathrm{M}-\mathrm{CH}_{2}-\mathrm{CO}_{2}\right]^{\cdot++}$ and 106.06473 $\left[\mathrm{M}-\mathrm{CH}_{2}-\mathrm{CHO}_{2}\right]^{\cdot+}$.

\section{1-[(2E,4E)-2,4-Decadienoyl]pyrrolidine (4)}

1-[(2E,4E)-2,4-Decadienoyl]pyrrolidine was detected on peak ion $\mathrm{m} / \mathrm{z} 221.17796$ and the formula $\mathrm{C}_{14} \mathrm{H}_{23} \mathrm{NO}$. The fragmentation on methyl group led the development ion $\mathrm{m} / \mathrm{z} 208.16921$ [M- $\mathrm{CH}]^{\circ+}$; the fragmentation on pyrrole ring led the development ion $\mathrm{m} / \mathrm{z} 137.09520$ [M- $\left.\mathrm{C}_{5} \mathrm{H}_{10} \mathrm{~N}\right]^{\cdot+}$ - the characteristic of $\mathrm{N}$-methylpyrrolidines; ion $\mathrm{m} / \mathrm{z}$ $123.07973\left[\mathrm{M}-\mathrm{CH}_{2}-\mathrm{C}_{5} \mathrm{H}_{10} \mathrm{~N}\right]^{\cdot+}$; and $109.06395\left[\mathrm{M}-\mathrm{C}_{2} \mathrm{H}_{4}-\mathrm{C}_{5} \mathrm{H}_{10} \mathrm{~N}\right]^{\cdot+}$. 


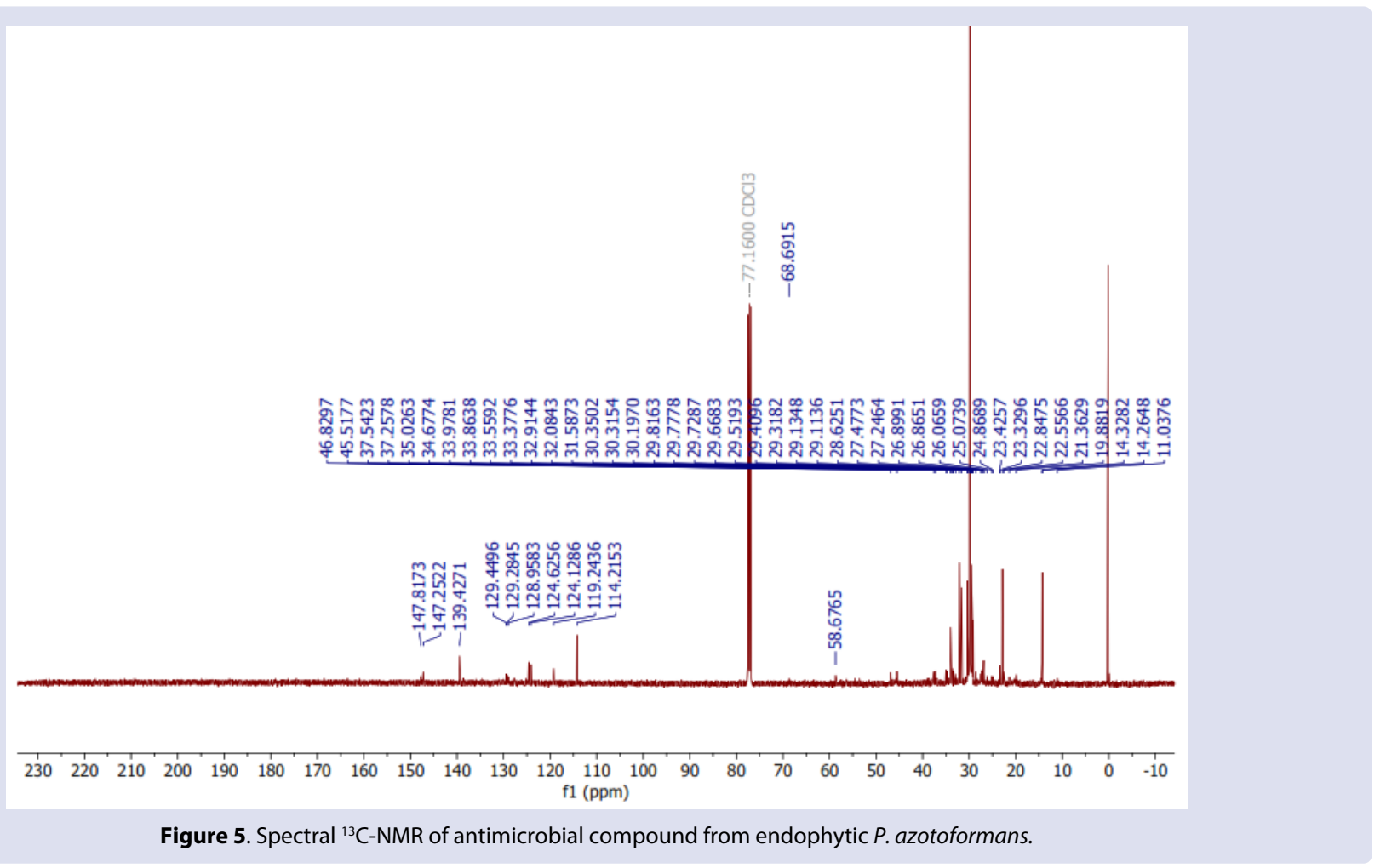

Table 4. Bioactive compound from fractions of P. azotoformans identified by LC-MS/MS.

\begin{tabular}{|c|c|c|c|c|c|c|c|c|c|}
\hline Fraction & $t_{R}$ & $\begin{array}{l}\mathrm{m} / \mathrm{z} \\
{[\mathrm{M}+\text { adduct }]}\end{array}$ & Adducts & $\begin{array}{l}\text { Neutral } \\
\text { mass }\end{array}$ & $\begin{array}{l}\text { Mass } \\
\text { error } \\
(\mathrm{mDa})\end{array}$ & $\begin{array}{l}\text { Fragmentation ions } \\
\text { (observed peak) }\end{array}$ & Formula & Compound & $\begin{array}{l}\text { Structure } \\
\text { number }\end{array}$ \\
\hline \multirow{3}{*}{ F1 } & 10.34 & 324.1569 & $+\mathrm{Na}$ & 301.16779 & -0.1 & $\begin{array}{l}290.17548 ; 288.19585 ; 286.18009 \\
272.16415 ; 149.05952 ; 122.05819\end{array}$ & $\mathrm{C}_{18} \mathrm{H}_{23} \mathrm{NO}_{3}$ & Futoamide & 1 \\
\hline & 10.21 & 260.1643 & $+\mathrm{H}$ & 259.15723 & -0.2 & $174.0547 ; 158.0597 ; 120.0439$ & $\mathrm{C}_{16} \mathrm{H}_{21} \mathrm{NO}_{2}$ & Candidate Mass 1 & \\
\hline & 10.24 & 276.1596 & $+\mathrm{H}$ & 275.15214 & 0.1 & $\begin{array}{l}174.0547 ; 158.0597 ; 120.0439 \\
135.06681 ; 134.09621 ; 133.08812\end{array}$ & $\mathrm{C}_{16}^{16} \mathrm{H}_{21}^{21} \mathrm{NO}_{3}^{2}$ & Candidate Mass 2 & \\
\hline \multirow{5}{*}{ F2 } & 9.79 & 150.0912 & $+\mathrm{H}$ & 149.08406 & -0.1 & $\begin{array}{l}120.08049 ; 107.07233 ; 106.06483 ; \\
94.06481 ; 92.05121\end{array}$ & $\mathrm{C}_{9} \mathrm{H}_{11} \mathrm{NO}$ & Gentialutine & 2 \\
\hline & 9.13 & 166.0861 & $+\mathrm{H}$ & 165.07898 & -0.2 & $\begin{array}{l}151.06236 ; 150.05470 ; 149.04724 ; \\
148.07529 ; 136.07425 ; 134.05973 \\
123.06763 ; 122.09581 ; 120.08035 ; \\
107.07164 ; 106.06473\end{array}$ & $\mathrm{C}_{9} \mathrm{H}_{11} \mathrm{NO}_{2}$ & Gentiatibetine & 3 \\
\hline & 9.88 & 400.2384 & $+\mathrm{H}$ & 399.23106 & 0.1 & $253.16971 ; 134.09621$ & $\mathrm{C}_{26} \mathrm{H}_{29} \mathrm{~N}_{3} \mathrm{O}$ & Candidate Mass 3 & \\
\hline & 9.03 & 283.1807 & $+\mathrm{H}$ & 282.17321 & 0.2 & $163.09435 ; 162.09116 ; 134.09596$ & $\mathrm{C}_{18} \mathrm{H}_{22} \mathrm{~N}_{2} \mathrm{O}$ & Candidate Mass 4 & \\
\hline & 10.31 & 311.1755 & $+\mathrm{H}$ & 310.16813 & 0.1 & $\begin{array}{l}296.29501 ; 176.07041 ; 162.09117 ; \\
148.07541\end{array}$ & $\mathrm{C}_{19} \mathrm{H}_{22} \mathrm{~N}_{2} \mathrm{O}_{2}$ & Candidate Mass 5 & \\
\hline \multirow{5}{*}{ F3 } & 9.98 & 244.1693 & $+\mathrm{Na}$ & 221.17796 & 2.2 & $159.06751 ; 146.05960$ & $\mathrm{C}_{14} \mathrm{H}_{23} \mathrm{NO}$ & $\begin{array}{l}\text { 1-[(2E,4E)-2,4-Decadienoyl] } \\
\text { pyrrolidine }\end{array}$ & 4 \\
\hline & 10.40 & 272.2006 & $+\mathrm{Na}$ & 249.20926 & 2.1 & 216.17409 & $\mathrm{C}_{16} \mathrm{H}_{27} \mathrm{NO}$ & Dihydrolycopodine & 5 \\
\hline & 10.31 & 270.1853 & $+\mathrm{Na}$ & 247.19361 & 2.5 & $232.16907 ; 232.16885 ; 218.15439$ & $\mathrm{C}_{16}^{16} \mathrm{H}_{25} \mathrm{NO}$ & Lycopodine & 6 \\
\hline & 10.58 & 300.2319 & $+\mathrm{H}$ & 299.22491 & -0.3 & $\begin{array}{l}298.21648 ; 198.09089 ; 184.07522 \\
172.07544 ; 159.06758 ; 130.06467\end{array}$ & $\mathrm{C}_{20} \mathrm{H}_{29} \mathrm{NO}$ & Candidate Mass 6 & \\
\hline & 10.64 & 326.2476 & $+\mathrm{H}$ & 325.24056 & -0.3 & $\begin{array}{l}\text { 184.07522; } 298.216 .48 ; 184.07522 ; \\
172.07544 ; 159.06758 ; 146.05974\end{array}$ & $\mathrm{C}_{22} \mathrm{H}_{31} \mathrm{NO}$ & Candidate Mass 7 & \\
\hline \multirow{5}{*}{ F4 } & 10.37 & 324.1568 & $+\mathrm{Na} ;+\mathrm{H}$ & 301.16779 & -0.2 & $\begin{array}{l}\text { 290.17392; 272.16476; 149.05938; } \\
122.05918\end{array}$ & $\mathrm{C}_{18} \mathrm{H}_{23} \mathrm{NO}_{3}$ & Futoamida & 1 \\
\hline & 10.24 & 270.1852 & $+\mathrm{Na}$ & 247.19361 & 2.4 & $232.16877 ; 192.13820$ & $\mathrm{C}_{16} \mathrm{H}_{25} \mathrm{NO}$ & Lycopodine & 6 \\
\hline & 10.27 & 276.1594 & $+\mathrm{H}$ & 275.15214 & 0.0 & $260.18519 ; 184.07529 ; 178.0497$ & $\mathrm{C}_{16}^{16} \mathrm{H}_{21}^{25} \mathrm{NO}_{3}$ & Candidate Mass 8 & \\
\hline & 10.42 & 304.1905 & $+\mathrm{H}$ & 303.18344 & -0.2 & $\begin{array}{l}298.21636 ; 286.18024 ; 184.07508 ; \\
160.03849 ; 107.08456\end{array}$ & $\mathrm{C}_{18} \mathrm{H}_{25} \mathrm{NO}_{3}$ & Candidate Mass 9 & \\
\hline & 10.51 & 324.2316 & $+\mathrm{H}$ & 323.22491 & -0.5 & 254.16918; 198.09032; 98.98333 & $\mathrm{C}_{22} \mathrm{H}_{29} \mathrm{NO}$ & Candidate Mass 10 & \\
\hline \multirow{5}{*}{ F5 } & 10.09 & 244.1695 & $+\mathrm{Na}$ & 221.17796 & 2.3 & $159.06742 ; 130.06480$ & $\mathrm{C}_{14} \mathrm{H}_{23} \mathrm{NO}$ & $\begin{array}{l}\text { 1-[(2E,4E)-2,4-Decadienoyl] } \\
\text { pyrrolidine }\end{array}$ & 4 \\
\hline & 5.43 & 227.1752 & $+\mathrm{H}$ & 226.16813 & -0.2 & $209.16420 ; 100.11142$ & $\mathrm{C}_{12} \mathrm{H}_{22} \mathrm{~N}_{2} \mathrm{O}_{2}$ & Cyclo-(Leu-Ile) & 7 \\
\hline & 10.27 & 272.2007 & $+\mathrm{Na}$ & 249.20926 & 2.2 & $159.06748 ; 149.02288$ & $\mathrm{C}_{16} \mathrm{H}_{27} \mathrm{NO}$ & Dihydrolycopodine & 5 \\
\hline & 10.35 & 322.0797 & $+\mathrm{Na}$ & 299.09061 & -0.2 & 236.97797; 225.07253 & $\mathrm{C}_{15} \mathrm{H}_{13} \mathrm{~N}_{3} \mathrm{O}_{4}$ & Candidate Mass 11 & \\
\hline & 10.48 & 457.2766 & $+\mathrm{Na}$ & 434.28797 & -0.5 & $317.19561 ; 155.06975$ & $\mathrm{C}_{22} \mathrm{H}_{42} \mathrm{O}_{8}$ & Candidate Mass 12 & \\
\hline
\end{tabular}




\section{Dihydrolycopodine (5) and Lycopodine (6)}

Dihydro-lycopodine was detected at ion $\mathrm{m} / \mathrm{z} 249.2096$ with formula $\mathrm{C}_{16} \mathrm{H}_{27} \mathrm{NO}$. The fragmentations on alcohol group and methyl group led the ion $\mathrm{m} / \mathrm{z} 216.17409\left[\mathrm{M}-\mathrm{CH}_{3}-\mathrm{H}_{2} \mathrm{O}\right]^{\cdot+}$. Lycopodine was detected at ion $\mathrm{m} / \mathrm{z} 247.19361$ with formula $\mathrm{C}_{16} \mathrm{H}_{25} \mathrm{NO}$. The fragmentation on methyl group led the ion $\mathrm{m} / \mathrm{z} 232.16907\left[\mathrm{M}-\mathrm{CH}_{3}\right]^{++}$; further fragmentation led development ion $\mathrm{m} / \mathrm{z} 218.15439\left[\mathrm{M}-\mathrm{C}_{2} \mathrm{H}_{5}\right]^{*+}$, and $192.13829[\mathrm{M}-$ $\left.\mathrm{C}_{4} \mathrm{H}_{7}\right]^{\cdot+}$.

The ${ }^{13} \mathrm{C}-\mathrm{NMR}$ on $\mathrm{F} 1$ with mixture compound condition (Figure 5) was exhibit aromatic signal (ppm) at $\delta \mathrm{C} 147.25(\mathrm{C})$ and $147.25(\mathrm{C})$. Signal between 110-130 $(\mathrm{CH})$ ppm might be aromatic signal or alkenes. Signal at 58.68 (C) ppm come from alkanes which bound with nitrogen, oxygen or halogen. The intense peak on the range at $30 \mathrm{ppm}$ revealed the aliphatic carbon of alkanes as $\mathrm{CH} 2$. Signal on area 5-45 ppm also might be carbon $\mathrm{sp} 3$. The ${ }^{13} \mathrm{C}-\mathrm{NMR}$ revealed the structure of compound (1) (Figure 6) with mixture of aliphatic carbon. The mixture of aliphatic carbon might come from fatty acid chain. However, there's no identified or detected signal on $170 \mathrm{ppm}$, hence the aliphatic carbon might come from mixture compounds.

\section{DISCUSSION}

The crude extract was more effective to inhibit C. albicans as yeast rather than bacteria. The antimicrobial activity differences might be related to the cell wall composition of microorganism and the antimicrobial compound itself. The major components of the C. albicans cell wall are fibrillar polysaccharides and protein. ${ }^{14}$ For bacteria like $S$. aureus, the major components of the cell wall are murein teichoic acid and protein. Murein consists of glycan strands, which are cross-linked by peptide bridges. ${ }^{15}$

The fractions able to go lower on inhibition concentration than the crude extract. However, the diameter clear zone resulted from fractions were in the range $1.00-4.00 \mathrm{~mm}$, which is considered low inhibition.

F1 containing compound futoamide (1), candidate mass 1 and candidate mass 2 . F1 has shown antimicrobial activity on the pathogenic microbes-on previous research. ${ }^{4}$ Futoamide (1) containing benzene ring attached with dioxol (benzodioxole), heptadiene as polyunsaturated fatty acid, and isobutyl amide. Grouped as alkaloamide or isobutyl amide alkaloid syntheses from L-lysine, futoamide was also found on family Piperaceae, with bioactive activity as antioxidant. Futoamide in Piper sp. is homolog to pellitorine, an insecticidal, antimycobacterial, anticandidal, and anti-plasmodial compound. ${ }^{16,17}$ Pellitorine was also found in bioactive compound of Pseudomonas stutzeri which act to inhibit Mycobacterium. Beside pellitorine, P. stutzeri also able to produce brachystamide B, guineensine, piperlonguminine, and retrofractamide, bioactive compound with benzodioxol group attached to unsaturated alkyl amide. ${ }^{18}$ The bioactive properties on futoamide lay on its alkyl amide structure and on its benzodioxole group. Benzodioxole as oxygen heterocyclic compound is an antibacterial and antifungal compound. ${ }^{19}$ Candidate mass 1 and 2 based on ion $\mathrm{m} / \mathrm{z}$ peaks are 2-heptil-3-hydroxyquinolone and 3-n-heptyl-3-hydroxy-1,2,3,4-tetrahydroquinoline2,4-dione, respectively. Alkyl-hydroxyquinolines frequently found on Pseudomonas spp. as quorum-sensing molecule. The compounds also have been identified on marine-derived Streptomyces sp., it has strong anticandidal than antibacterial effect. It has an ability to inhibit hyphal growth of C. albicans. ${ }^{20} \mathbf{F 1}$ also has strong anticandidal activity than its antibacterial activity. ${ }^{4}$ Therefore, this identification compound supported that F1 has a potential as anticandidal compound.

F2 consist of compound gentialutine (2), gentiatibetine (3), candidate mass 3, candidate mass 4 and candidate mass 5. Gentialutine (2) and gentiatibetine (3) are monoterpenoid alkaloid ${ }^{13}$ containing pyridine nucleus, synthesis from nicotinic acid. Pyridine ring has an ability as antibacterial activity. The potential lays on a proton-accepting nitrogen atom (heterocyclic or non-heterocyclic) and one or more hydrogen proton donating hydrogen atom. ${ }^{21}$ The pyridine ring on gentialutine fused with cyclo-pentane, and on gentiatibentine, it's fused with pyran ring. Gentialutine (2) first isolated from root of Gentiana lutena and leaf of Menyanthes trifoliata. ${ }^{22}$ But, there wasn't any report to be produced by Pseudomonaceae, the similar compound of cyclo-pentane pyridine has been reported to be isolated from marine fungi (Wallemia sebi) and it has weak antibacterial against Enterobacter. ${ }^{23}$ Thus, also reflected on our research where F2 only inhibit $S$. aureus ATCC 6583 on $1,000 \mu \mathrm{g} / \mathrm{mL}$ and gave negative result for others microbes. Several<smiles>CC(C)(C)NC(=O)/C=C/CC/C=C/c1ccc2c(c1)OCO2</smiles>

A

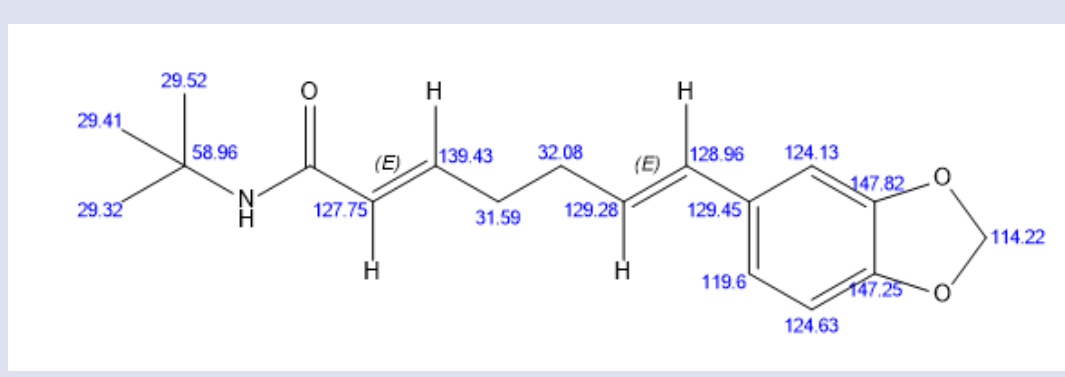

Figure 6. The structure of futoamide from endophytic P. azotoformans: prediction (A) and based on result (B). 
report on gentiatibentine (3) was identified on ethyl acetate extracted of Streptomyces with ability against Plasmodium parasite ${ }^{24}$ and from methanol extract of Melophlus sarasinorum (sponge marine) with ability as antioxidant. ${ }^{25}$ But, there hasn't any report on gentiabentine antimicrobial activity, pyranopyridines as an antibacterial compound has been reported with mechanism interrupting the efflux pump on E. coli. ${ }^{26}$

F3 have several compounds like 1-[(2E,4E)-2,4-Decadienoyl] pyrrolidine (4), dihydro-lycopodine (5), lycopodine (6), candidate mass 6 , and candidate mass 7 . The 1-[(2E,4E)-2,4-Decadienoyl]pyrrolidine (4) is an alkaloid compound, containing pyrrolidine nucleus synthesis from L-ornithine, and unsaturated fatty acid. ${ }^{13,17}$ It also known as sarmentine, mostly found in Piper sp. ${ }^{13,16}$ It has potency as herbicide ${ }^{16}$ and antimicrobial compound. ${ }^{27}$ Based on research methanol extract of Piper sarmentosun that contain sarmentine, it was able to inhibit Mycobacterium tuberculosis, Methicillin Resistant Staphylococcus aureus (MRSA), S. aureus, E. coli and P. aeruginosa. ${ }^{18,27}$ From our previous research, $\mathbf{F} 3$ also have antibacterial and anticandidal activity. ${ }^{4}$ The interesting study was reported on cytotoxic activity of sarmentine, but indeed its activity was still lower than taxol as positive control. ${ }^{17}$ From our observation, there hasn't any publication on sarmentine from Pseudomonaceae. The similar ring of pyrrolidine compound was isolated from Pseudomonas sp. as $\mathrm{N}$-(decanoyl)pyrrolidine and $\mathrm{N}$-(decenoyl)pyrrolidine with antimicrobial activity against several microbe. $^{28}$

Dihydrolycopodine (5) and lycopodine (6) grouped as Lycopodiumalkaloid, it was first isolated on moses Lycopodiaceae..$^{29,30}$ The multicyclic of lycopodine biosynthesis arise from acetoacetyl co-A and L-lysine. The lycopodium alkaloid has been well explained regarding the source, classes, and potency. It's also mentioned the characteristic of lycopodium alkaloid on acetylcholinesterase inhibition activity. ${ }^{30}$ As for its bioactivity, lycopodine and dihydrolycopodine from Lycopodium clavatum extracted with chloroform-alcohol has been discovered to have antioxidant, antiviral, and antimicrobial activity against E. coli, P. aeruginosa, Proteus mirabilis, Acinobacter baumannii, Klebsiella pneumoniae, S. aureus, B. subtilis, C. albicans, and C. parapsilosis. ${ }^{31}$ Thus, strengthen our result from previous research on $\mathbf{F} 3$, on its ability as anticandidal. Lycopodine has been discovered on endophytic fungus Paraboremia from Lycopedium serratum ${ }^{32}$, but the bioactive activities has not been explored, yet. The candidate compound 6 based on ion $\mathrm{m} / \mathrm{z}$ fragmentation is 2-decyl-1-methyl-quinolin-4-one. It was also reported as antimicrobial compound from endophytic marine Pseudomonas sp. ${ }^{33}$

F4 consist of futoamida (1), lycopodine (6), candidate mass 8, candidate mass 9 , and candidate mass 10 . There hasn't any single publication on lycopodine isolated from Pseudomonaceae. However, pelletierine as lycopodine intermediet was also found on P. azotoformans. ${ }^{34}$ Therefore, further studies were needed to support this finding.

F5 consist of compound (4), cyclo-(leu-ile) (7), dihydro-lycopodine (5), candidate mass 11 , and candidate mass 12 . The cyclo-(leu-ile) (7) or 3-butan-2-yl-6-(2-methylpropyl)piperazine-2,5-dione is a diketopiperazine, grouped as cyclo-dipeptide (CDP) alkaloid. CDP can be produced by bacteria, fungi and plants. CDP synthesis by enzyme NRPS. CDP has bioactivities as antioxidant, antiviral, antitumor, antifungal and antibacterial ${ }^{18,35,36}$, and plant growth regulation. ${ }^{37}$ The reported CDP from Pseudomonas were cyclo-(leu-pro), cyclo(val-pro), cyclo-(pro-tyr), and cyclo-(meth-pro). ${ }^{18}$ Some CDP in Pseudomonas are involved in quorum sensing that mediated plant growth. ${ }^{36}$ Cyclo-(leu-ile) has been reported to be identified on marine Bacillus subtilis ${ }^{38}$, and chiral as cyclo-(ile-leu) was identified on fungus Verruculina enalia ${ }^{39}$ but its bioactivity has not been explored, yet. From our exploration, there's no cyclo-(leu-ile) has been reported to be isolated from Pseudomonas. CDP has potential as antimicrobial, for instance cyclo-(arg-pro) from marine Pseudomonas sp. that has been developed as antibiotic. ${ }^{40}$
Alkaloids on the microorganism play a role as inhibitors compound for competitors, thus functionally as antimicrobial compound. Alkaloid soluble on non-polar solvent like chloroform and also soluble on alcohol. ${ }^{21}$ Alkaloid soluble on water at acidic condition and soluble on lipid at neutral and basic condition. Thus characteristic, give ability on alkaloid to pass membrane in the neutral form and ability to be transported in protonated from. Several alkaloid containing nitrogen and/or oxygen heterocyclic that rich with electron therefore it can act as proton acceptor and donor, and forming interaction like hydrogen bound, dipol-dipol interaction, van der Waals, and $\pi$-stacking interaction with biomolecular target like enzyme and receptor. ${ }^{8}$ Thus ability explaining the antimicrobial mechanism on alkaloid, rather it is specific interaction with specific biomolecular target (ligand binding) or non-specific interaction (electrostatic and hydrophobic interaction) ${ }^{41,42}$

Antimicrobial mechanism of alkaloid like inhibition of sortase, inhibition of biofilm formation and inhibition of secretion system. Alkaloid able to interrupt protein synthase of cell wall thus lead the rupture on the cell wall and disrupt the turgor of cytoplasm. Alkaloid with low molecular weight might be able to diffuse into cell, and attenuated the influx-efflux mechanism in cytoplasm that led the cell lysis. But if the cell able to increase the cell efflux to pump out the alkaloid, the cell will be proceed to maintain the cell integrity. ${ }^{9,21} \mathbf{F} 4$ and $\mathbf{F} 5$ has better inhibition on S. typhimurium and E. coli, than any others bacteria. Aside efflux pump, alkaloid like cyclo-dipeptide might be attacking pore on the membrane cell bacteria by cationic-anionic affinity of amino group. Several alkaloid like quinolone have antiquorum sensing to others bacteria and attenuated electron transport mechanism. ${ }^{21,37}$ Alkaloid mechanism of action on C. albicans were due to inhibition of filament formation and the disruption of biofilm formation that prevent the adhesion of C. albicans to target (antiadhesin), and C. albicans cell wall components. Alkaloid with aliphatic chain able to diffuse into C. albicans cell. ${ }^{41,42}$

From identification of crude extract and the fractions, synergism effect might apply on the antimicrobial compound of endophyte $P$. azotoformans. Synergism effect were found on different class of natural product. Anticandidal natural product might have synergism effect ${ }^{41}$, as several antimicrobial which combinate the antimicrobial mechanism to strengthen the inhibition or to prevent increasing risk infection by others pathogen. ${ }^{42}$

Regarding from the publication on $P$. azotoformans gene cluster on secondary metabolite that consist of 4 NRPS gene cluster, 3 bacteriocin gene cluster, 1 terpen gene cluster and 1 unspecified gene cluster ${ }^{43}$ thus reflected on the antimicrobial compound identified in this research. The expression of secondary metabolite might be influenced by environment factors. This result describes the extraction and elucidates the identified antimicrobial compound of $P$. azotoformans, which can be further explored for the potency on medicinal and industrial area.

\section{CONCLUSION}

P. azotoformans strain UICC B-91 has potency as antimicrobial compound with range of minimum inhibition was $1,000-125 \mu \mathrm{g} /$ $\mathrm{mL}$. Anticandidal activities on $P$. azotoformans extracts were on synergism effect. Antimicrobial compounds have been discovered from P. azotoformans UICC B-91 as alkaloid on CHON arrangements with molecular weight approximately 100-450 Da. The identified alkaloid group are isobutyl amide alkaloid, monoterpenoid alkaloid, lycopodium alkaloid and cyclo-dipeptide alkaloid.

\section{ACKNOWLEDGEMENT}

This research was supported by Research Grant of DRPM Kemenristek BRIN-Kemendikbud Ristek-LLDIKTI3 awarded to RHP number 1867/E4/AK.04/2021. The authors acknowledge the facilities, scientific 
and technical support from Advanced Characterization Laboratories Serpong, National Research and Innovation Institute through ELayanan Sains, Badan Riset dan Inovasi Nasional. We also would like to thank to Microbiology Laboratory at Center of Chemical and Packaging, Jakarta and Research Center for Biomaterial, Indonesian Institute of Sciences (LIPI), Cibinong for valuable technical assistance.

\section{REFERENCES}

1. Pratiwi $\mathrm{RH}$, Hidayat I, Hanafi M, Mangunwardoyo W. Identification and characterization of three endophytic bacteria from Neesia altissima (Malvaceae) antagonistic to diarrhea-causing bacteria. Malays J Microbiol. 2016;12(4):300-307. doi:10.21161/mjm.81515

2. Quattrocchi U. CRC World Dictionary of Medicinal and Poisonous Plants: Common Names, Scientific Names, Eponyms, Synonyms, and Etymology. New York: CRC Pres; 2016. https://books.google. co.id/books? id=-37OBQAAQBAJ\&dq=Neesia+altissima+medicinal +plant\&source=gbs_navlinks_s. Accessed June 12, 2020.

3. Khare E, Mishra J, Arora NK. Multifaceted Interactions Between Endophytes and Plant: Developments and Prospects. Front Microbiol. 2018;9(November):1-12. doi:10.3389/fmicb.2018.02732

4. Oktarina E, Pratiwi RH, Mangunwardoyo W, Hidayat I, Saepudin E. In vitro antimicrobial activities of several extracts endophytic Pseudomonas azotoformans UICC B-91. IOP Conf. Series: Earth and Environmental Science. 2021; 948 (2021) 012068. doi:10.1088/17551315/948/1/012068

5. Tedesco P, Maida I, Esposito FP, et al. Antimicrobial activity of monoramnholipids produced by bacterial strains isolated from the Ross Sea (Antarctica). Mar Drugs. 2016;14(83):1-14. doi:10.3390/ md14050083

6. Lee DS, Eom SH, Jeong SY, et al. Anti-methicillin-resistant Staphylococcus aureus (MRSA) substance from the marine bacterium Pseudomonas sp. UJ-6. Environ Toxicol Pharmacol. 2013;35(2):171-177. doi:10.1016/j.etap.2012.11.011

7. França $P$ de. Avaliação Da Atividade Antimicrobiana De Bactérias Associadas À Invertebrados Marinhos Oriundos Da Antártica. XX Alam. 2016:785

8. Kerru N, Gummidi L, Maddila S, Gangu KK, Jonnalagadda SB. A review on recent advances in nitrogen-containing molecules and their biological applications. Molecules. 2020;25(8). doi:10.3390/ molecules25081909

9. Yan Y, Li X, Zhang C, Lv L, Gao B, Li M. Research progress on antibacterial activities and mechanisms of natural alkaloids: A review. Antibiotics. 2021;10(3). doi:10.3390/antibiotics10030318

10. Pratiwi $\mathrm{RH}$, Hidayat I, Hanafi M, Mangunwardoyo W. Antibacterial compound produced by Pseudomonas aeruginosa strain UICC B-40, an endophytic bacterium isolated from Neesia altissima. J Microbiol. 2017;55(4):289-295. doi:10.1007/s12275-017-6311-0

11. Mandryk MN, Kolomiets El, Dey ES. Characterization of antimicrobial compounds produced by Pseudomonas aurantiaca S-1. Polish J Microbiol. 2007;56(4):245-250.

12. Dewanjee S, Gangopadhyay M, Bhattacharya N, Khanra R, Dua TK. Bioautography and its scope in the field of natural product chemistry. J Pharm Anal. 2015;5(2):75-84. doi:10.1016/j.jpha.2014.06.002

13. Buckingham J, Baggaley KH, Roberts AD, Szabo LF. Dictionary of Alkaloids. second edi. London: CRC Press; 2010. doi:10.1016/03788741(90)90073-3

14. Chaffin WL. Candida albicans Cell Wall Proteins. Microbiol Mol Biol Rev. 2008;72(3):495-544. doi:10.1128/mmbr.00032-07

15. Romaniuk JAH, Cegelski L. Bacterial cell wall composition and the influence of antibiotics by cell-wall and whole-cell NMR. Philos Trans R Soc B Biol Sci. 2015;370(1679). doi:10.1098/rstb.2015.0024

16. Stöhr JR, Xiao PG, Bauer R. Constituents of Chinese Piper species and their inhibitory activity on prostaglandin and leukotriene biosynthesis in vitro. J Ethnopharmacol. 2001;75(2-3):133-139. doi:10.1016/S0378-8741(00)00397-4
17. Kumar V, Bhatt V, Kumar N. Amides From Plants: Structures and Biological Importance. In: Ur Rahman A, ed. Studies in Natural Products Chemistry. Vol 56. 1st ed. Amsterdam, Netherlands: Elsevier B.V.; 2018:287-333. doi:10.1016/B978-0-444-64058-1.00009-1

18. Zeng $X$, Zhang $P, H e$, et al. NPASS: natural product activity and species source database for natural product research, discovery and tool development. Nucleic Acids Res. 2018;4(46).

19. Phi Thi D, Doan Thi Mai H, Cao DD, et al. Novel 1,3-Benzodioxole From Marine-Derived Actinomycete in East Vietnam Sea. Nat Prod Commun. 2020;15(5):1-6. doi:10.1177/1934578X20920042

20. Kim H, Hwang JY, Chung B, et al. 2-Alkyl-4-hydroxyquinolines from a Marine-Derived Streptomyces sp. Inhibit Hyphal Growth Induction in Candida albicans. Mar Drugs. 2019;17(133):10.

21. Cushnie TPT, Cushnie B, Lamb AJ. Alkaloids: An overview of their antibacterial, antibiotic-enhancing and antivirulence activities. Int J Antimicrob Agents. 2014;44(5):377-386. doi:10.1016/j. ijantimicag.2014.06.001

22. Duke JA. Handbook of Phytochemical Constituents of GRAS Herbs and Other Economic Plants. Boca Raton, FL.: CRC Press.; 1992.

23. Xu L, Meng W, Cao C, Wang J, Shan W, Wang Q. Antibacteria and antifungal compounds from marine fungi. Mar Drugs. 2015;13(6):3479-3513. doi:10.3390/md13063479

24. Damayanti E, Lisdiyanti $P$, Sundowo A, et al. Antiplasmodial activity, biosynthetic gene clusters diversity, and secondary metabolite constituent of selected indonesian Streptomyces. Biodiversitas. 2021;22(6):3478-3487. doi:10.13057/biodiv/d220657

25. Sahidin I, Sabandar CW, Wahyuni, et al. Investigation of Compounds and Biological Activity of Selected Indonesian Marine Sponges. Nat Prod J. 2020;10(3):312-321. doi:10.2174/2210315509666190627105237

26. Opperman TJ, Kwasny SM, Kim HS, et al. Characterization of a novel pyranopyridine inhibitor of the AcrAB efflux pump of Escherichia coli. Antimicrob Agents Chemother. 2014;58(2):722 733. doi:10.1128/AAC.01866-13

27. Nurul', Sanusi A, Adawiyah Umar R, et al. Chemical Compositions and Antimicrobial Properties of Piper Sarmentosum-A Review. IOSR J Dent Med Sci e-ISSN. 2017;16(8):62-65. doi:10.9790/0853-1608066265

28. Asolkar R, Lucia A, Todd C. Control of phytopathogenic microorganisms with pseudomonas $\mathrm{sp}$. and substances and compositions derived therefrom. https://worldwide.espacenet. com/patent/search/family/049083253/publication/WO2013130680 A1?q=pn\%3DWO2013130680A1. Published 2013.

29. Olafsdóttir ES, Halldorsdottir ES, Pich NM, Omarsdottir S Lycopodium Alkaloids: Pharmacology. In: K. IR, JM. M, eds. Natural Products. Berlin: Springer Berlin Heidelberg; 2013. doi:https://doi. org/10.1007/978-3-642-22144-6_42

30. Ma X, Gang DR. The Lycopodium alkaloids. Nat Prod Rep. 2004;21.

31. Orhan I, Özçelik B, Aslan S, et al. Antioxidant and antimicrobia actions of the clubmoss Lycopodium clavatum L. Phytochem Rev. 2007:6(1):189-196. doi:10.1007/s11101-006-9053-x

32. Ishiuchi K, Hirose D, Suzuki T, et al. Identification of Lycopodium Alkaloids Produced by an Ultraviolet-Irradiated Strain of Paraboeremia, an Endophytic Fungus from Lycopodium serratum var. longipetiolatum. J Nat Prod. 2018;81(5):1143-1147. doi:10.1021/ acs.jnatprod. $7 \mathrm{~b} 00627$

33. Bultel-Poncé V, Berge JP, Debitus C, Nicolas JL, Guyot M. Metabolites from the sponge-associated bacterium Pseudomonas species. Mar Biotechnol. 1999;1(4):384-390. doi:10.1007/PL00011792

34. KEGG. Tropane, piperidine and pyridine alkaloid biosynthesis Reference pathway - Pseudomonas azotoformans. https://www. genome.jp/pathway $/$ mapno=00960\&category_type $=$ species\&cate gory=Pseudomonas+azotoformans. Published 2019.

35. Wang $X$, Li Y, Zhang X, Lai D, Zhou L. Structural diversity and biological activities of the cyclodipeptides from Fungi. Molecules. 2017;22(12). doi:10.3390/molecules22122026 
36. Mishra AK, Choi J, Choi SJ, Baek KH. Cyclodipeptides: An overview of their biosynthesis and biological activity. Molecules. 2017;22(10):1-13. doi:10.3390/molecules22101796

37. Kimura Y, Sawada A, Kuramata M, et al. Brevicompanine C, cyclo(D-Ile-L-Trp), and cyclo-(D-Leu-L-Trp), plant growth regulators from Penicillium brevi-compactum. J Nat Prod. 2005;68(2):237-239. doi:10.1021/np040178p

38. Lu $X$, Shen $Y$, Zhu $Y$, et al. Diketopiperazine constituents of marine Bacillus subtilis. Chem Nat Compd. 2009;45(2):290-292. doi:10.1007/s10600-009-9270-9

39. Lin $Y, W u X$, Deng $Z$, et al. The metabolites of the mangrove fungus Verruculina enalia No. 2606 from a salt lake in the Bahamas. Phytochemistry. 2002;59(4):469-471. doi:10.1016/S00319422(01)00470-8
40. Blunt JW, Munro MHG. Dictionary of Marine Natural Products. London: CRC Press.; 2008.

41. Zida A, Bamba S, Yacouba A, Ouedraogo-Traore R, Guiguemdé RT Anti-candida albicans natural products, sources of new antifungal drugs: A review. J Mycol Med. 2017;27(1):1-19. doi:10.1016/j. mycmed.2016.10.002

42. Martin $\mathrm{H}$, Kavanagh $\mathrm{K}$, Velasco-Torrijos $\mathrm{T}$. Targeting adhesion in fungal pathogen Candida albicans. Future Med Chem. 2021;13(3):313-334. doi:10.4155/fmc-2020-0052

43. Fang $Y, W u$ L, Chen G, Feng G. Complete genome sequence of Pseudomonas azotoformans S4, a potential biocontrol bacterium. J Biotechnol. 2016;227:25-26. doi:https://doi.org/10.1016/j. jbiotec.2016.04.020

\section{GRAPHICAL ABSTRACT}
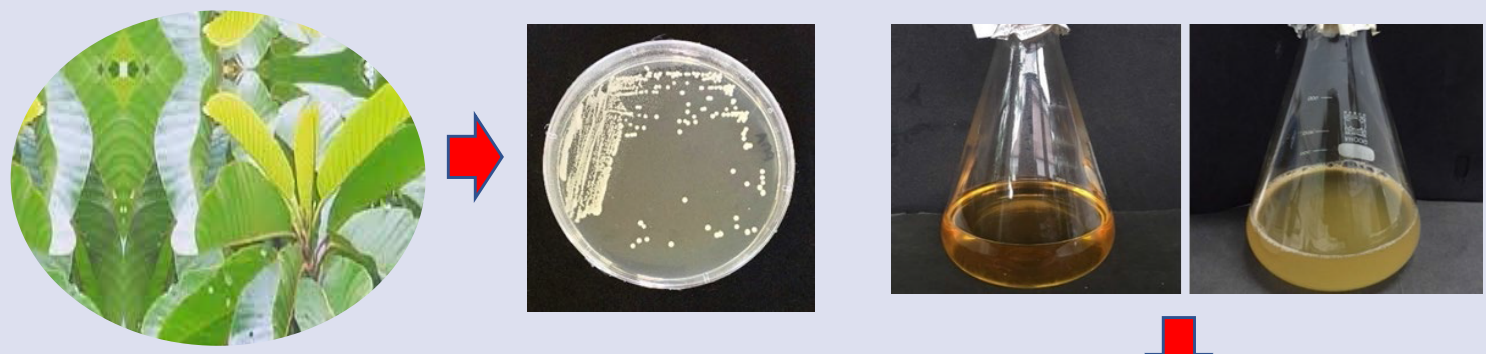

\section{RESULT:}

Detected several compound as

Futoamide

(C18H23NO3), Gentialutine (C9H11NO), Gentiabetine (C9H11NO2), 1-[(2E,4E)-2,4decadienoyl]pyrrolidine (C14H23NO), Lycopodine (C16H25NO), and Dihydro-lycopodine (C16H27NO)

Fractionation and

\section{Spectroscopy}

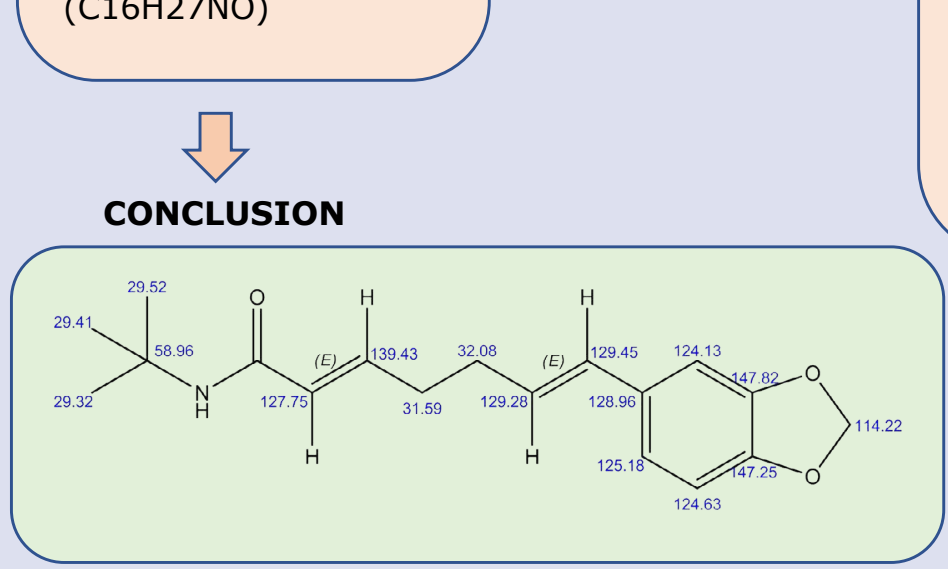

Antimicrobial activity against Escherichia coli ATCC 8739, Bacillus cereus ATCC 10876, Staphylococcus aureus ATCC 6583, Staphylococcus aureus ATCC 25923, Salmonella typhimurium ATCC 25241, Pseudomonas aeruginosa ATCC 15442, Bacillus subtilis ATCC 19659 and Candida albicans ATCC 10231 


\section{ABOUT AUTHORS}
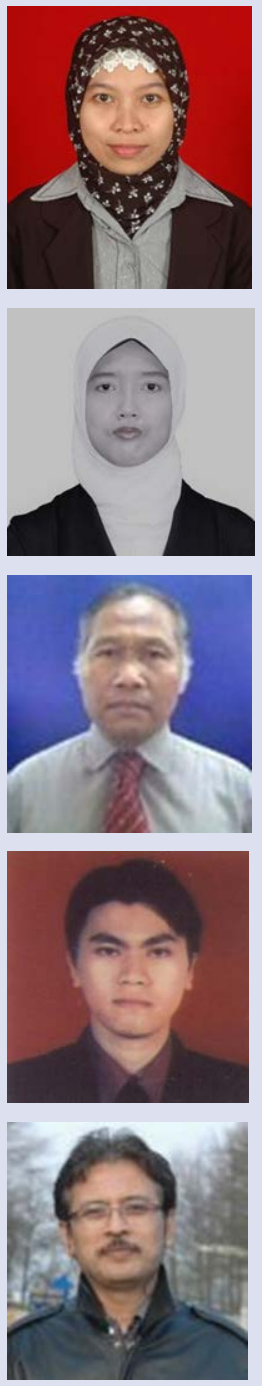

Rina Hidayati Pratiwi is a Associate Professor and Lecturer at Department of Mathematics and Natural Sciences Education, Faculty of Post Graduated and Department of Biological Education, Universitas Indraprasta PGRI, Jakarta 12530, Indonesia. She has research experience in the field of Microbiology and bioactive compound from microorganisms, phage, drug discovery, and in silico research of bioactive compounds.

Eva Oktarina is a Master Student at Master Program of Chemistry, Faculty of Mathematics and Natural Sciences, Universitas Indonesia, Depok, West Java 16424, Indonesia. She works at Center of Chemical and Packaging, Jakarta.

Wibowo Mangunwardoyo is a Professor and Lecturer at Department of Biology, Faculty of Mathematics and Natural Sciences, Universitas Indonesia, Depok, West Java 16424, Indonesia. He has research experience in the area of Microbiology and Biotechnology.

Iman Hidayat is a Researcher and Head of Research Center for Biomaterial, Indonesian Institute of Sciences (LIPI), Cibinong, Indonesia, Indonesia. He has research experience in the field of Mycology, and Biosystematics of microorganism.

Endang Saepudin is a Senior Lecturer at Department of Chemistry, Faculty of Mathematics and Natural Sciences, Universitas Indonesia, Depok, West Java 16424, Indonesia. He has research experience in the field of bioactivities of naturally accuring compounds.

Cite this article: Pratiwi RH, Oktarina E, Mangunwardoyo W, Hidayat I, Saepudin E. Antimicrobial Compound from Endophytic Pseudomonas azotoformans UICC B-91 of Neesia altissima (Malvaceae). Pharmacogn J. 2022;14(1): 172-181. 